\title{
Orpheus: Automatic Composition System Considering Prosody of Japanese Lyrics
}

\author{
Satoru Fukayama ${ }^{1}$, Kei Nakatsuma ${ }^{1}$, Shinji Sako ${ }^{2}$, Yuichiro Yonebayashi ${ }^{1}$, \\ Tae Hun Kim ${ }^{1}$, Si Wei Qin ${ }^{1}$, Takuho Nakano ${ }^{1}$, Takuya Nishimoto ${ }^{1}$, \\ and Shigeki Sagayama ${ }^{1}$ \\ 1 The University of Tokyo \\ \{fukayama, k-nakatsuma, yonebayashi, kim, qin, t-nakano, \\ nishi, sagayama\}@hil.t.u-tokyo.ac.jp \\ 2 Nagoya Institute of Technology, \\ sako@mmsp.nitech.ac.jp
}

\section{Song Composition Algorithm Considering Prosody}

We present an algorithm for song composition using prosody of Japanese lyrics. Since Japanese is a "pitch accent" language, listener's apprehension is strongly affected by the pitch motions of the speaker. For example, the meaning of Japanese word "ha-shi" changes with the pitch. It means "bridge" with an upward pitch motion, and "chopsticks" with the motion inversed. A melody attached to the lyrics cause an effect similar to the pitch accent. Therefore we can assume that pitches of Japanese lyrics give constraints on pitch motions of the melody. Furthermore, chord progression, rhythm and accompaniment give constraints on the transitions and occurrences of the melody notes. If a certain melody for the lyrics were obtained, the melody would satisfy these constraints. Conversely, we can compose a song by finding the melody which optimally meets the condition.

\section{Implementation and Experimental Results}

Orpheus is an automatic composition system that we implemented using melody composition algorithm based on prosody. This system computes melody from the lyrics input with choices of chord progressions, rhythm patterns, and accompaniment instruments. We used Galatea-Talk 4 text-to-speech engine to analyze the prosody of Japanese lyrics, and HMM singing voice synthesizer [5] to generate the vocal part. We also implemented the system as a web-based application 1 .

We did two experiments to evaluate the system. Firstly, we asked a classical music composer to evaluate 59 generated songs in five-grade evaluation. Secondly, we uploaded our system to get comments from a large number of users on the internet. During a year of operation, about 56,000 songs were generated by the users and 1378 people answered the questions about Orpheus and the generated songs. The results are shown in Fig. 11 and Fig. 21 Judging from the results, about $70.8 \%$ commented that the generated songs are attractive, and $84.9 \%$ of the users had fun trying this system.

\footnotetext{
${ }^{1}$ http://orpheus.hil.t.u-tokyo.ac.jp/automatic-composition/index.en.cgi
} 


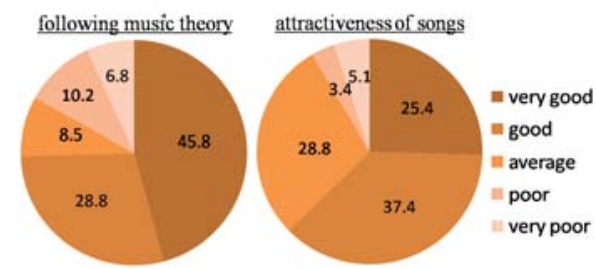

Fig. 1. Evaluation results on 59 songs by a classical music composer [\%]

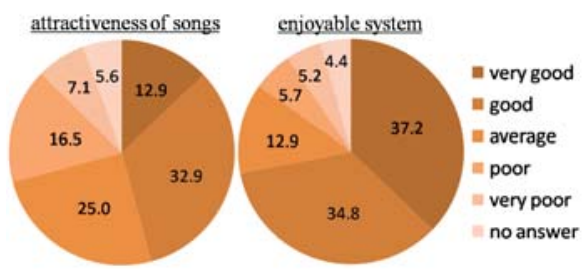

Fig. 2. Evaluation results on generated songs and the Orpheus by 1378 users [\%]

\section{Discussions and Conclusion}

This research attempted to design an algorithm to compose a song automatically from the lyrics using prosody information, which enables users to make their original songs easily. The results indicate that our method and implemented system Orpheus is an enjoyable solution for amateur musicians.

However, it should be noted that our algorithm can be applied to lyrics written in "pitch accent" languages only. As a next step, we plan to extend the composition algorithm to handle "stress accent" languages, such as English, by putting constraints on metric structure of the melody.

Acknowledgments. We would like to thank Hitomi Kaneko for the evaluation of generated songs and useful discussions from the composer's point of view. This research was funded by the Crest Muse Project [6].

\section{References}

1. Hiller, L., Isaacson, L.: Experimental Music. McGraw-Hill, New York (1959)

2. Xenakis, I.: Formalized Music, Revised edn. Pendragon Press, New York (1992)

3. Cope, D.: Computers and Musical Style. A-R Editions. Madison, Wisconsin (1991)

4. Galatea Project, http://hil.t.u-tokyo.ac.jp/ galatea/

5. Sako, S., Miyajima, C., Tokuda, K., Kitamura, T.: A Singing Voice Synthesis System Based on Hidden Markov Model. Transactions of IPSJ, 719-727 (2004) (in Japanese)

6. CrestMuse Project, http://www.crestmuse.jp/index-e.html 